[Aus der Univ.-Augenklinik zu Leipzig (Direktor: Geheimer Rat Prof. Dr. Sattler) und der Univ.-Kinderklinik zu Leipzig (Direktor Prof. Dr. Thiemich).]

\title{
Experimenteller Beitrag zur Ätiologie der Keratomalacie.
}

\author{
Von \\ M. Goldschmidt, \\ Assistent der Augenklinik. \\ Mit 4 Figuren im Text.
}

Bis vor wenigen Jahren war man allgemein der Ansicht, dass eine aus Eiweiss, Fett, Kohlehydraten, Salzen und Wasser in entsprechenden Verhältnissen zusammengesetzte und in ausreichender Menge verabfolgte Nahrung zum Leben und zur Entwicklung des tierischen Organismus genüge. Diese Auffassung ist in neuerer Zeit erschüttert worden, und man neigt auf Grund einer Reihe klinischer und experimenteller Beobachtungen der Anschauung zu, dass es ausser den oben genannten Stoffen noch andere Substanzen geben müsse, ohne die die Erhaltung des Lebens auf die Dauer nicht möglich sei. Die Untersuchungen, die zu dieser Auffassung geführt haben, galten der Erforschung der Genese einiger Krankheiten, von denen man zum Teil schon lange vermutet hatte, dass sie in irgendeiner Beziehung zur Ernährung ständen, nämlich Skorbut, Möller-Barlow sche Krankheit (infantiler Skorbut) and Beri-Beri.

Es war schon längst bekannt, dass der Skorbut des Menschen bei langdauerndem Mangel an frischen Vegetabilien aufzutreten pflegte. Holst and Fröhlich(1) gelang es, diese Erkrankung experimentell an Meerschweinchen zu erzeugen durch ausschliessliche Verfütterung von Hafer und andern Getreidearten. Durch Zusatz von nur $1 \mathrm{~g}$ frischen Kohls pro Tag zur Getreidekost konnte der Krankheit vorgebeugt werden. Auch Zusatz frischer Milch verhütete den Aussbruch des Skorbuts; gekochte Milch dagegen erwies sich als wirkungslos.

Die Möller-Barlow sche Krankheit, der Skorbut der Kinder, entsteht bei ausschliesslichem Genuss von Milch, die zu lang- 
dauerndem oder zu hohem Erhitzen ausgesetzt gewesen (Milchkonserven), Mehlpräparaten usw., Zusatz von frischen Fruchtsäften oder in jüngster Zeit von alkoholischem Extrakt von Futterrüben [Frei s e (2) ] zur krankmachenden Nahrung heilt die Krankheit in kurzer Zeit.

Eine Hauptstütze der Auffassung von dem Vorhandensein bisher nicht berücksichtigter lebenswichtiger Stoffe in der Nahrung stellen die Ergebnisse der klinischen und experimentellen Studien zur Klärung der Beriberigenese dar. Es hat sich als wahrscheinlich erwiesen, dass die Beriberi durch den Mangel an Substanzen entsteht, die im Perikarp des Reiskorns vorhanden sind. Denn die ausschliessliche Ernährung mit poliertem Reis, wie sie besonders in Japan vorkommt, ruft die genannte Erkrankung hervor, während die einseitige Verfütterung von unpoliertem Reis sich als unschädlich erweist. Eykman (3), der als erster das Beriberiproblem experimentell angriff, konnte durch Verfütterung von geschältem Reis an Geflügel ein Krankheitsbild erzeugen, das der menschlichen Beri-Beri sehr ähnlich ist (Polyneuritis gallinarum). Durch Zusatz von Reiskleie oder deren wässerigem Extrakt konnte er den Ausbruch der Krankheit verhüten. Tiere, die ungeschälten Reis erhielten, erkrankten überhaupt nicht.

Weitere wichtige Resultate wurden an Untersuchungen über das Wachstum erzielt. Hopkins (4) konnte nachweisen, dass bei Ernährung mit kalorisch ausreichenden, richtig zusammengesetzten, aber sorgfältig gereinigten Gemischen von Eiweiss, Fett, Kohlehydrat, Salzen und Wasser das Wachstum der weissen Ratten ausbleibt, und dass es nur des Zusatzes einer sehr geringen Menge ungekochter Milch $(1-4 \%$ der Trockensubstanz der Standardkost) bedarf, um das Wachstum zu ermöglichen. Statt Milch konnte er auch Gemüsesaft oder Haferextrakt verwenden. (Osborne und Mendel (5) konnten übrigens trotz sorgfältiger Reinigung des Nahrungsgemischs eine Wachstumszunahme erzeugen.) Ste p p (6) hat endlich gezeigt, dass bei ausschliesslicher Verfütterung einer durch Alkohol und Äther extrahierten Nahrung Mäuse zugrunde gehen. Durch Zusatz von Alkohol-Ätherextrakt aus Eigelb oder Kalbshirn zu der vorher ungenügenden Nahrung konnten die Tiere am Leben erhalten werden; Zusatz von Butter oder reinem Lezithin war wirkungslos.

Die chemische Charakterisierung der Stoffe, mit denen wir es hier zu tun haben, ist von Casimir Funk (7), Suzuki, Shi- 
mamura und Odake (8) versucht worden. Ersterer betrachtet eine organische Base als Träger der genannten Wirkungen (Vitamin), letztere haben ein kristallinisches Pikrat aus Reiskleie dargestellt (Oryzanin).

Bei Versuchen, die sich mit ähnlichen Ernährungsproblemen befassen und die zurzeit an der Leipziger Kinderklinik von Freise und Frank an Ratten ausgeführt werden, zeigte sich bei einer grösseren Anzahl von Versuchstieren eine Augenerkrankung, die mit der Art der Ernährung in Zusammenhang gebracht werden konnte. Die Affektion ist, wie aus den später mitzuteilenden Protokollen und dem Ergebnis der histologischen Untersuchung hervorgeht, als eine experimentelle Keratomalacie der Ratten aufzufassen. Ob und welche Schlüsse daraus auf die menschliche Keratomalacie gezogen werden können, soll weiter unten erörtert werden.

Von jeher hat die Frage nach der Ätiologie der Keratomalacie das Interesse der Ophthalmologen in besonderem Masse in Anspruch genommen. Mit den Fortschritten der allgemeinen Pathologie wechselten auch die Anschauungen über diese Frage. Schon vor längerer Zeit hat man einen Zusammenhang zwischen Ernährungsstörung und Keratomalacie feststellen können [F örster (9) u. a.]. In der bakteriologischen Ära geriet diese Anschauung mehr in den Hintergrund und man fasste die Keratomalacie in erster Linie als eine Infektion auf, für die verschiedene Erreger verantwortlich gemacht wurden (Xerosebazillen, Pneumokokken, Lueserreger). Erst in neuerer Zeit sieht man wieder in Ernährungsstörungen, speziell dem Mehlnährschaden, den Hauptfaktor zum Zustandekommen dieser Erkrankung.

\section{Experimenteller Teil.}

$\mathrm{Zu}$ sämtlichen Versuchen wurden weisse Ratten genommen, die von drei Elternpaaren abstammten. Die Ratten, auf die sich unsere experimentelle Erfahrung stützt, rührten aus der 3. bis 5. Generation her. Da das Bild der menschlichen Keratomalacie an ein bestimmtes Altersoptimum gebunden ist, war es nötig, die Versuche an Tieren verschiedenen Alters durchzuführen. Es wurden deshalb Ratten im Anfangsgewicht von 40-160g, also lauter noch im Wachstum begriffene Tiere gewählt. Die Ratten wurden nach Hopkins ausschliesslich mit folgendem chemisch-reinen Nahrungsgemisch ernährt: 
Experimenteller Beitrag zur Ätiologie der Keratomalacie.

Casein Hammarsten

22,0

Stärke $1 / 2$ Std. lang mit Alkohol beiss extrahiert 42,0

Rohrzucker

21,0

Schweinefett auf $140^{\circ} 1 \mathrm{Std}$. lang erwärmt 12,4

Asche aus Hundekuchen und Hafermehl (Salze) 2,6

Von diesem Gemisch frassen die Ratten durchschnittlich $5-6 \mathrm{~g}$ pro Tag. Ausserdem erhielten sie gek och tes destilliertes Wasser. Bei dieser Versuchsanordnung traten bei einer Reihe von Tieren charakteristische Augensymptome auf. Im einzelnen verliefen die Versuche folgendermassen:

Ratten vom Anfangsgewicht bis ungefähr $100 \mathrm{~g}$.

Ratte 28.

6. II. Beginn des Versuchs. Gewicht $45 \mathrm{~g}$.

28. II. Haarausfall an den Lidern.

8. III. Gewicht $46 \mathrm{~g}$. Beiderseits Enophthalmus.

15. III. Gewicht $46 \mathrm{~g}$. Beginnende Tribung der Hornhaut.

26. III. R. Hornhaut total matt, wie mit Fett bestrichen, nasal kleine Infiltrationen. L. gleicher Befund.

Ratte wird zur histol. Untersuchung getötet. Gewicht $44 \mathrm{~g}$.

Ratte 1.

6. II. Beginn des Versuchs. Gewicht $42 \mathrm{~g}$.

23. II. Beginn des Gewichtsabfalls. Gewicht $49 \mathrm{~g}$.

3. III. Haarausfall an den Lidern. Enophthalmus. Gewieht $45 \mathrm{~g}$.

8. III. Trübung der Hornhäute. Gewicht $42 \mathrm{~g}$.

19. III. Hornhäute total matt und undurchsichtig. Gewicht $39 \mathrm{~g}$.

26. III. Ulzeröser Zerfall beider Hornhäute. Irisprolaps. Gewicht $31 \mathrm{~g}$,

Zweeks histol. Untersuchung getötet.

Ratte 36 .

28. III. Beginn des Versuchs. Anfangsgewicht $81 \mathrm{~g}$.

Mitte IV. Gewicht $84 \mathrm{~g}$. Beginn des Gewichtsabfalls. Haarausfall, Enophthalmus.

26. IV. Hormhäute trocken. Gewicht $67 \mathrm{~g}$.

28. IV. Beiderseits Mattigkeit. Gewicht $69 \mathrm{~g}$.

2. V. R. beginnendes Uleus unten und nasenwärts. L. matte Hornhaut. Gewicht $69 \mathrm{~g}$.

6. V. R. totale Einschmelzung. L. beginnendes Uleus. Gewicht $66 \mathrm{~g}$. Exitus.

\section{Ratte 37.}

28. III. Beginn des Versuchs. Anfangsgewicht $84 \mathrm{~g}$. wicht $91 \mathrm{~g}$.

Mitte IV. Beginn des Gewichtsabfalls, Haarausfall, Enophthalmus. Ge-

24. IV. Beginnende Trübung der Hornhäute. Gewicht $78 \mathrm{~g}$. 
28. IV. Hormhäute total matt. Gewicht $81 \mathrm{~g}$.

2. V. R. Hornhaut total eingeschmolzen. L. total matt. Gewicht $82 \mathrm{~g}$. 10. V. Getötet zwecks histologischer Untersuchung. Gewicht $81 \mathrm{~g}$.

\section{Ratte 29.}

28. III. Beginn des Versuchs, Anfangsgewicht $87 \mathrm{~g}$.

Mitte IV. Beginn des Gewichtsabfalls. Haarausfall, Enophthalmus. Gewicht $99 \mathrm{~g}$.

23. IV. Xerosis beiderseits. Gewicht $87 \mathrm{~g}$.

28. IV. Beiderseits Uleera comeae. Gewicht $82 \mathrm{~g}$.

2. V. Beiderseits totale Einschmelzung. Gewicht $78 \mathrm{~g}$.

7. V. Exitus. Gewicht $67 \mathrm{~g}$.

Ratte 45.

28. III. Beginn des Versuchs. Anfangsgewicht $93 \mathrm{~g}$.

5. IV. Beginn des Gewichtsabfalls. Gewicht $105 \mathrm{~g}$.

Mitte IV. Enophthalmus. Haarausfall. Gewicht ungefähr $86 \mathrm{~g}$.

28. IV. Beginnende Trubung beiderseits. Gewicht $82 \mathrm{~g}$.

2. V. R. sehr starke Trübung. L. total eingesehmolzene Iris, Linse vorgefallen. Gewicht $78 \mathrm{~g}$.

6. V. R. Ulcus, links status idem. Gewicht $76 \mathrm{~g}$.

9. V. Exitus. Gewicht $58 \mathrm{~g}$.

\section{Ratte 46.}

28. III. Beginn des Versuchs. Anfangsgewicht $102 \mathrm{~g}$.

4. IV. Beginn des Gewichtsabfalls. Gewicht $118 \mathrm{~g}$.

Mitte IV. Haarausfall, Enophthalmus. Gewicht ungefăhr $100 \mathrm{~g}$.

28. IV. Beginnende Trubung beiderseits. Gewicht $98 \mathrm{~g}$.

2. V. Beiderseits Ulcera. Gewicht $89 \mathrm{~g}$.

6. V. Beiderseits totale Einschmelzung. Gewicht $90 \mathrm{~g}$.

9. V. Status idem. Gewieht $81 \mathrm{~g}$.

12. V. Exitus. Gewicht $70 \mathrm{~g}$.

Aus den angeführten Protokollen geht hervor, dass nach anfänglichem Gewichtsanstieg um ungefähr 8-12\% des Anfangsgewichts in der 2.-3. Woche ein Gewichtsabfall erfolgt, der meist bis zum Tode anhält. Der Gewichtsverlust beträgt zwischen 10 und $46 \%$ des Höchstgewichts. Ungefähr zur Zeit des Beginns des Gewichtsabfalls, also etwa in der 3. Woche, tritt das erste Augensymptom auf: A usfall der Lidbehaarung (ohne allgemeinen Haarausfall). Es folgt in der 3.-4. Woche ein sehr auffälliger Enophthalmus und erst in der 5.-6. Woche eine sichtbare Affektion der Hornhaut, nämlich Trockenheit, raschfolgende Trübung und ulzeröser Zerfall ohne stark entzündliche Erscheinungen. Die Tiere zeigen im übrigen einen verminderten Bewegungsdrang. 
- Ratten mit starkem Gewichtsabfall weisen im allgemeinen ein schwereres Krankheitsbild in bezug auf die Augenaffektion auf wie Ratten mit langsam abfallender Kurve. Die beiden Augen desselben Tieres sind öfters verschieden schwer ergriffen; immer aber erfolgt zuletzt der ulzeröse Zerfall, falls die Tiere nicht vorher eingehen oder getötet werden. Nie kommt es zu einer spontanen Besserung oder Heilung.

In weiteren Versuchen konnten wir nachweisen, dass es gelingt, Tiere, die bei der oben angegebenen Ernährung die beschriebene Hornhautaffektion akquirieren, zu heilen, sobald die nicht vollwertige Nahrung durch Zufuhr der lebenswichtigen Substanzen ergänzt wird. Der Zusatz bestand in unsern Versuchen aus $2 \mathrm{~cm}$ roher Magermilch, $d . h$. einer Menge, die nur 1-4\% der Trockensubstanz der Gesamtnahrung ausmacht und dem Grundfutter bezüglich seiner Zusammensetzung (Eiweiss; Fett, Kohlehydrate) durchaus entspricht. Diese Mehrzufuhr von täglich $2 \mathrm{ccm}$ Magermilch kommt, wie Hopkins (4) in seiner Arbeit Factors of diet ausführlich diskutiert, in kalorischer Hinsicht nicht in Frage.

Zu unsern therapeutischen Versuchen wurden solche Tiere verwandt, bei denen experimentell an einem Auge eine schwere typische Hornhautaffektion hervorgerufen war, während das andere Auge sich im Frühstadium der Erkrankung befand (deutliche Trübung, eventuell geringe Infiltration, immer Enophthalmus).

\section{Ratte 38 .}

28. III. Beginn des Versuchs. Gewicht $92 \mathrm{~g}$.

12. IV. Beginn des Gewichtsabfalls. Gewicht $105 \mathrm{~g}$.

23. IV. Ausfall der Lidhaare, Enophthalmus. Hornhaut o. B. Gewicht $89 \mathrm{~g}$. 28. IV. Beiderseits trockene Hornhaut. Gewicht $90 \mathrm{~g}$.

2. V. R. Hornhaut etwas getrübt. L. Trockenheit, Gewicht $84 \mathrm{~g}$.

6. V. Beiderseits Hornhant getrübt. Gewicht $80 \mathrm{~g}$.

9. V. R. Nasal Uleus. L. Trübung. Gewicht $79 \mathrm{~g}$.

10. V. Zusatz von $2 \mathrm{ecm}$ roher Mileh pro Tag. Gewieht $78 \mathrm{~g}$.

13. V. R. Hornhant total eingeschmolzen. L. geringe Mattigkeit. Enophthalmus. Gewieht $81 \mathrm{~g}$.

15. V. R. Geschwürfläche in Reinigung begriffen. L. Hornhaut glänzend, geringer Enophthalmus. Gewieht $90 \mathrm{~g}$.

18. V. R. beginnende Narbenbildung. L. Enophthalmus nicht mehr vorhanden. Gewicht $98 \mathrm{~g}$.

21. V. R. Phthisis bulbi. L, vollständig normaler Befund. Gewicht $101 \mathrm{~g}$.

25. V. Beiderseits status idem. Gewieht $109 \mathrm{~g}$.

1. VI. Status idem. Gewicht $120 \mathrm{~g}$. 
8. VI. Status idem. Gewicht $123 \mathrm{~g}$.

21. VI. Ratte nimmt dauernd an Gewicht zu.

Ratte 39.

28. III. Beginn des Versuchs. Gewicht $109 \mathrm{~g}$.

4. IV. Beginn des Gewichtsabfalls. Gewicht $119 \mathrm{~g}$.

15. IV. Enophthalmus. Haarausfall an den Lidern. Gewicht $94 \mathrm{~g}$.

28. IV. Leichte Trübung beider Hornhäute. Gewicht $100 \mathrm{~g}$.

2. V. Beiderseits geringe Mattigkeit. Gewicht $92 \mathrm{~g}$.

6. V. Status idem. Gewicht $91 \mathrm{~g}$.

9. V. R. Ulens. L. temporal stecknadelspitzgrosses Infiltrat, Gewicht $88 \mathrm{~g}$.

10. V. Zusatz von $2 \mathrm{ecm}$ roher Milch zur tägliehen Ration von $6 \mathrm{~g}$ Hopkinseher Nahrung. Gewicht $80 \mathrm{~g}$.

11. V. Gewicht $77 \mathrm{~g}$.

15. V. R. totale Einschmelzung der Hornhaut ohne stark entzündliche Erscheinungen. L. Infiltrat kaum mehr wahrnehmbar, Mattigkeit der Hornhaut geringer, Enophthalmus zurüekgegangen. Gewicht $87 \mathrm{~g}$.

18. V. R. Narbenbildung. L. Hornhaut erscheint normal. Kein Enophthalmus mehr. Gewicht $93 \mathrm{~g}$.

21. V. Status idem. Gewicht $105 \mathrm{~g}$.

25. V. Status idem. Gewicht $116 \mathrm{~g}$.

1. VI. Phthisis bulbi. L. normal. Gewicht $127 \mathrm{~g}$.

8. VI. Status idem. Gewicht $138 \mathrm{~g}$.

15. VI. Gewicht $146 \mathrm{~g}$.

Ratte nimmt dauernd weiter zu.

Ratte 38 und 39 zeigen in gleicher Weise wie die Ratten der früheren Versuche das Auftreten einer Hornhauterkrankung, und zwar auf der einen Seite im Anfangsstadium, auf der andern im Stadium der Einschmelzung. Das schwer affizierte Auge kann durch Zusatz von $2 \mathrm{ccm}$ roher Magermilch zu der gleichbleibenden Menge Hopkins nicht mehr beeinflusst werden und verfällt der Phthisis bulbi. Das andere wird wieder völlig normal. Zugleich steigt die Gewichtskurve steil an.

Histologiseher Befund: Bei der beginnenden Hornhautaffektion Fig. 1 und 2 (Ratte 28) besteht Verhornung und teilweise Abhebang der oberen Epithelschichten. Kerne der mittleren Epithellagen gequollen, hell, schlecht färbbar. Zylinderzellenlage gelockert, zellig infiltriert. Das Hornhautstroma zeigt an Stellen, die makroskopisch nieht infiltriert zu sein scheinen, geringe Infiltration. In den oberen und mittleren Sehichten neugebildete Gefässe, vom Limbus her bis in das Zentrum der Hornhaut reichend. Die makroskopisch bereits infiltrierten Stellen zeigen Auflockerung und ödematöse Durchtränkung des Hornhantstromas mit Aufhebung der regelmässigen Anordnung der Hornhautfasern. Reichliche zellige Infiltration, besonders in den oberen und mittleren Stromaschichten. Deseemet und Endothel intakt. Keine Bakterien.

In schweren Fällen, Figur 3 und 4 (Ratte 1), alle histologischen 
Experimenteller Beitrag zur Ätiologie der Keratomalacie.

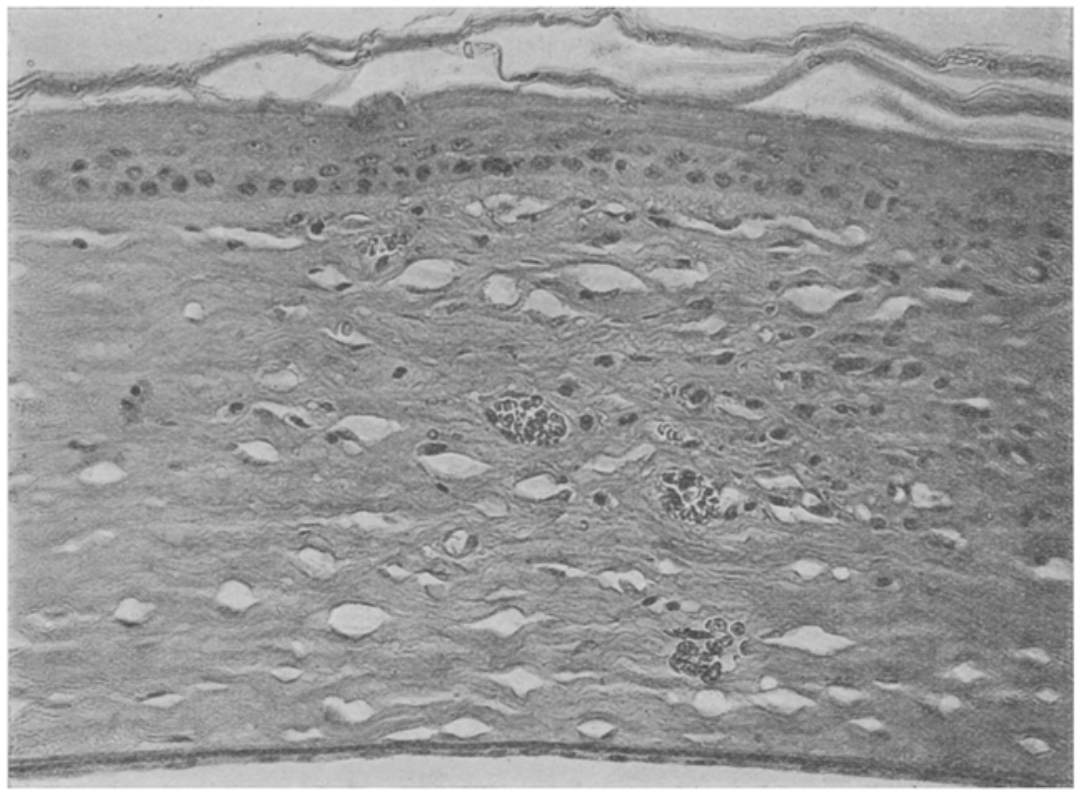

Fig. 1.

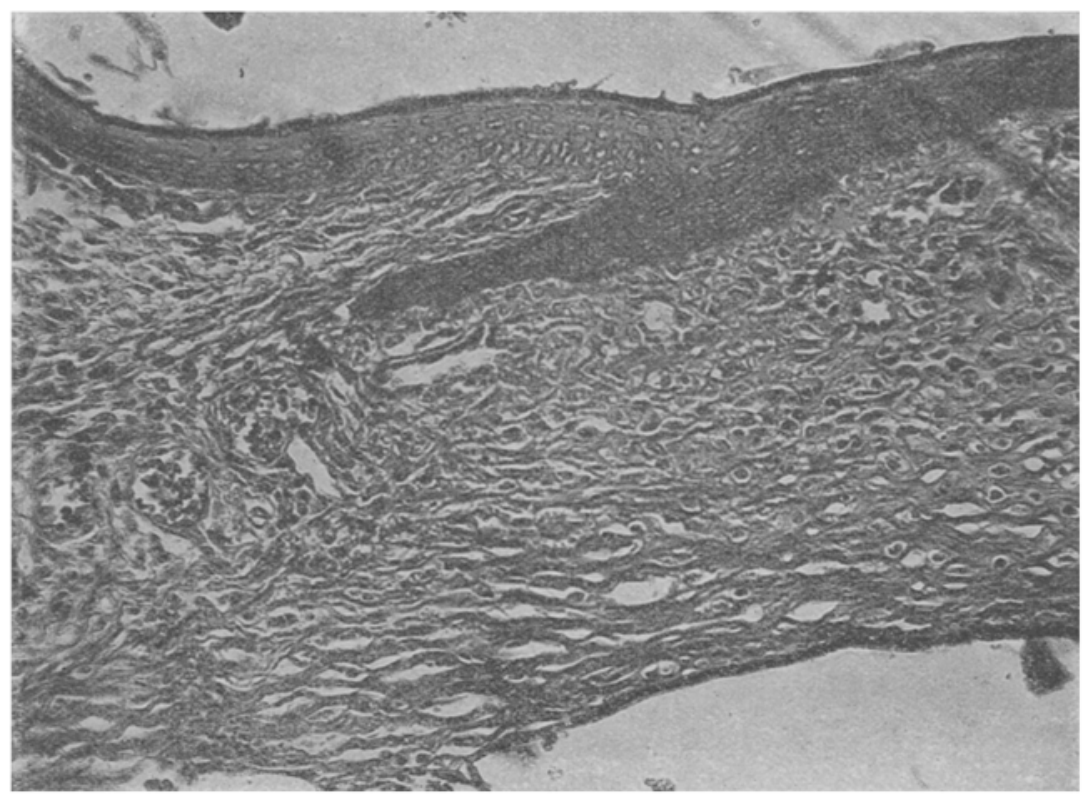

Fig. 2.

Zeichen des perforierten Ulcus. Ausgedehnte Nekrosen der Hornhaut. Hornhaut in grosser Ausdehnung leukozytär infiltriert. Längs dem Limbus e1- 


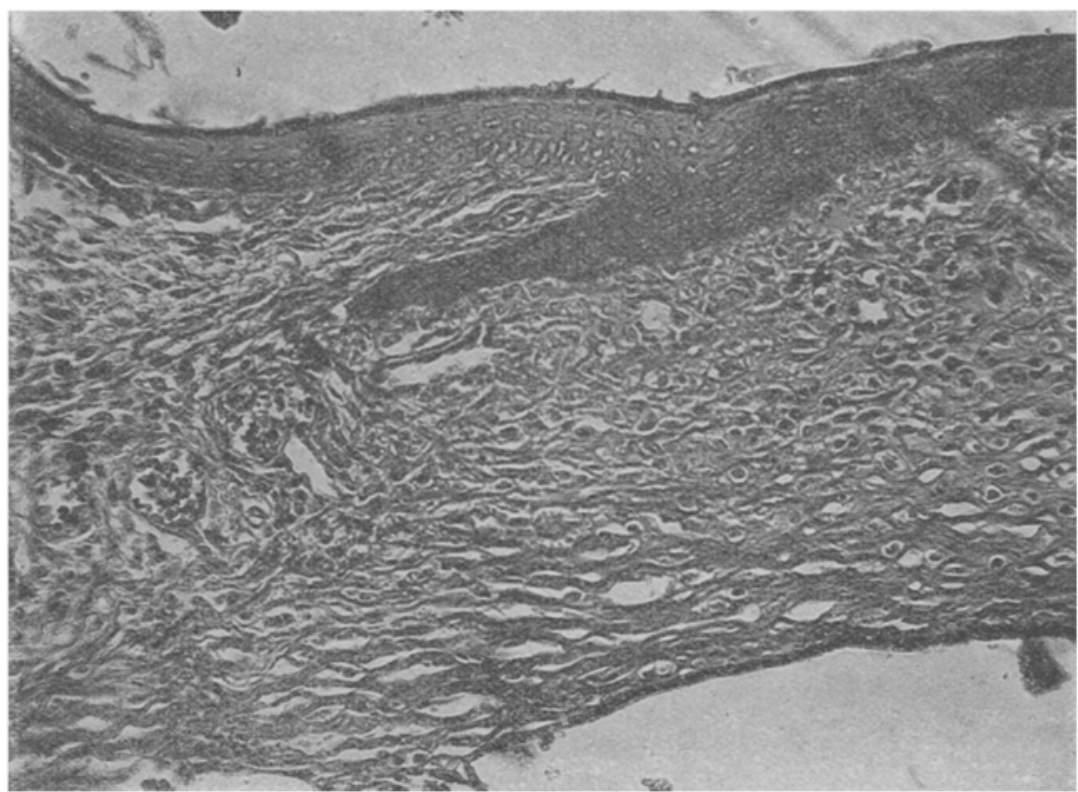

Fig. 3.

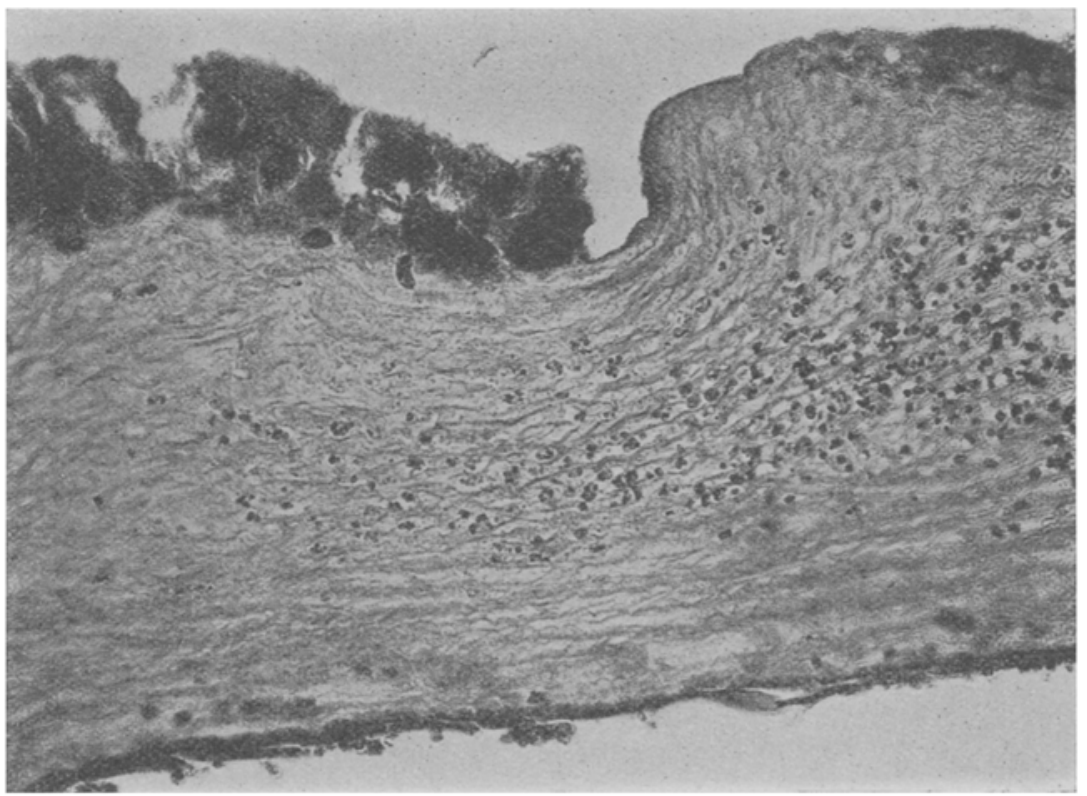

Fig. 4.

strecken sich vom Epithel ausgehende zapfenartige Wucherungen tief in das episklerale Bindegewebe hinein. Massenhaft Bakterien. 
Man könnte die beschriebene Augenaffektion auf eine allgemeine, nicht nur partielle Unteremährung zurückführen wollen. Dagegen spricht die Tatsache, dass in unsern Versuchen 38 und 39 die Tiere unter Zurückbildung der Augensymptome von dem Augenblick an stark an Gewicht zunehmen, als ihnen bei sonst gleicher Standardkost eine geringe, aber an den oben erwähnten Substanzen reiche Zulage, nämlich ungekochte Milch dargereicht wird. Es ist ausgeschlossen, dass dieser Zusatz, nach seinem kalorischen Wert bemessen, den rapiden Gewichtsanstieg zu bewirken imstande ist. Denn die Ratte 39 (Ratte 38 verhielt sich ähnlich) nahm in der Zeit, als sie an ungekochter Milch auf das Trockengewicht bezogen, insgesamt $1,36 \mathrm{~g}$ Zulage erhielt, im ganzen $40 \mathrm{~g}$ an Körpergewicht zu. Welche Bestandteile dabei zum Ansatz kommen und wie weit Wasserretention eine Rolle spielt, soll hier nicht weiter erörtert werden. Das Auftreten und Verschwinden des Enophthalmus spricht für Bewegungen im Wasserhaushalt.

Mit Sicherheit konnte ausgeschlossen werden, dass es sich um eine Endemie handele. Denn normal ernährte Ratten, die den mit Hornhautulzeration behafteten dauernd zugesetzt waren, erkrankten niemals.

Ein weiterer Punkt von grosser Bedeutung sind die anscheinend auch im Tierexperiment nachweisbaren Beziehungen der Affektion zum Al te r der Ratten. Von unsern Versuchstieren zeigten alle diejenigen im Gewicht von ungefähr 40-100 g die charakteristischen Erscheinungen; unter den Ratten rom Anfangsgewicht 100-120 g traten bei einigen trotz Gewichtsabfalls keine Augensymptome auf, und von den Tieren über $120 \mathrm{~g}$ zeigte kein einziges irgendwelche Augenerscheinungen. Entsprechend ihrer Bedeutung wird diese Teilfrage augenblicklich an einem grösseren Material weiter untersucht.

Nach dem geschilderten klinischen Verlauf und gestützt auf den histologischen Befund, der mit den Untersuchungen von Dötsch (10) über die menschliche Keratomalacie übereinstimmt, können wir diese Augenerkrankung als eine experimentelle Keratomalacie der Ratten auffassen. Es liegt nun nahe, die experimentellen Ergebnisse der Rattenkeratomalacie zu verwerten, um einen Einblick in die Ätiologie der menschlichen Keratomalacie zu erhalten. Wir hätten es demgemäss bei der menschlichen Keratomalacie, gleichgültig durch welche Ernährungsstörung her- 
vorgerufen und durch welche komplizierende Organerkrankung erschwert, in erster Linie mit einer partiellen Un terernährung zu tun. Betrachten wir beispielsweise den Mehlnährschaden unter diesem Gesichtspunkt, so ist hervorzuheben, dass die Mehle, insbesondere die industriellen Mehle, sehr arm an den oben erwähnten akzessorischen lebenswichtigen Substanzen sind. Es würde also schon allein von diesem Standpunkt aus die ausschliessliche Mehlnahrung als eine nicht vollwertige anzusehen sein, ganz abgesehen von der übrigen unzweckmässigen Zusammensetzung. Werden nun den auf dieser Basis an Keratomalacie erkrankten Kindern die fehlenden Substanzen in Form der Brustnahrung verabfolgt, so sieht man, auch nach den Erfahrungen an unserer Klinik, in einem grossen Teil nicht allzu weit vorgeschrittener Fälle unter Besserung des Allgemeinbefindens ein Zurückgehen der Augenerscheinungen mit entsprechenden reparativen Vorgängen. Diese Besserung scheint sich im wesentlichen nicht so sehr auf die Milch als zweckmässig zusammengesetztes Nahrungsgemisch von Eiweiss, Fett, Kohlehydraten und Salzen, sowie auf das Aufhören der einseitigen Kohlehydraternährung zu beziehen, als vielmehr auf die $\mathrm{Zufuhr}$ vorher fehlender Bestandteile. Dass es nicht die Milch als solche ist, die die Heilwirkung hervorruft, geht aus verschiedenen Beobachtungen hervor, die erkennen lassen, dass eine in bezug auf den Gehalt an diesen Stoffen nicht vollwertige Milch sogar. im Gegenteil die Krankheitserscheinungen hervorrufen kann. Thalberg (11) berichtet, dass er die meisten seiner Keratomalaciefälle bei Brustkindern gesehen habe, die durch blutarme oder infolge langen Fastens herabgekommene Mütter gestillt wurden. „Namentlich schien letzteres den Anstoss für das Auftreten einer Hornhautgangrän bei einem schon kranken Kinde abzugeben, auch wenn die Mutter angab, hinreichend Milch in der Brust zu haben, denn es häufte sich die Zahl der Erkrankungsfälle am Ende der Fastenzeit, während sonst trotz der bedeutenden Anzahl atrophischer Kinder einer von der armen Bevölkerung stark besuchten Ambulanz nur hin und wieder eine einseitige Hornhautgangrän vorkam." Eine Analogie findet diese Tatsache im Auftreten der Beriberi bei Säuglingen, die von beriberikranken Müttern genährt werden; auch kann die Erkrankung bereits auftreten, wenn bei der stillenden Frau noch keine Beriberisymptome festzustellen sind [Hirota(12)]. Es fehlen eben der an sich 
zweckmässigen Nahrung lebensnotwendige Bestandteile. Werden dieselben den Kindern in Form von Reiskleie, Milch einer gesunden Frau, selbst Kuhmilch zugeführt, so genesen die Kinder in kurzer Zeit. Eine Verarmung und ebenso eine nachfolgende Anreicherung des Organismus an diesen lebenswichtigen Substanzen macht sich also, insbesondere beim wachsenden Individuum, ziemlich rasch and in sehr tiefgreifender Weise geltend, was bei der ausserordentlichen Wirksamkeit der Stoffe nicht überraschend ist:

Über die Rolle, die diese für das Leben anscheinend unentbehrlichen Stoffe im Zellstoffwechsel spielen, lassen sich zurzeit nur Vermutungen aussprechen. Wahrscheinlich ist es, dass diese Substanzen für den Mineralstoffwechsel bedeutsam sind, indem sie die $\mathrm{Ca}$-Retention ermöglichen. In jüngster Zeit hat $\mathrm{Erd-}$ mann (13) in einem Vortrag über Veränderungen am Kaninchenauge im Gefolge von Ernährungsstörungen über ein Krankheitsbild berichtet, das mit dem von uns an Ratten erzielten grosse Ähnlichkeit besitzt. Er bezieht die Ursache dieser Erkrankung auf den Mangel an Kalk, d. h. Ca. In dem mir zu Gebote stehenden Referat fehlen leider nähere Angaben über die Zusammensetzung der Nahrung. Ein direkter $\mathrm{Ca}$-Mangel des Organismus ist bei unsern Versuchen durch die ganze Anordnung auszuschliessen. Immerhin ist es auf Grund der Vorstellungen, die man sich über die Wirkungsweise dieser Substanzen machen kann, nicht unverständlich, dass man auch durch Aufhebung der $\mathrm{Ca}$-Zufuhr ein dem von uns erzeugten ähnliches Krankheitsbild hervorrufen kann. Näheres Eingehen auf die Arbeit Erdmanns müssen wir uns bis nach erfolgter ausführlicher Publikation derselben vorbehalten.

\section{Zusammenfassung.}

Es gelingt bei weissen Ratten bis zum Gewicht von ungefähr $100 \mathrm{~g}$ durch partielle Unterernährung ein Krankheitsbild zu erzeugen, das klinisch wie auch pathologisch-anatomisch als Keratomalacie aufzufassen ist. Die Krankheitserscheinungen werden zum Schwinden gebracht durch Zusatz von $2 \mathrm{ccm}$ roher Magermilch, deren Wirkung nicht an das Vorhandensein von Eiweiss, Fett, Kohlehydraten, Salzen gebunden ist, sondern auf dem Gehalt an noch unbekannten, aber für das Leben notwendigen Substanzen beruht. Es ist wahrscheinlich, dass die Keratomalacie der Ratten ihrer Entstehung nach zu einer Reihe 
366 M. Goldschmidt, Experimenteller Beitrag zur Ätiologie der Keratomalacie.

von Krankheitsbildern zu zählen ist, der auch experimenteller Skorbut, experimentelle Beriberi angehören.

Klinische, experimentelle und pathologisch-anatomische Analogien zwischen Rattenkeratomalacie und menschlicher Keratomalacie machen es wahrscheinlich, dass auch bei der menschlichen Keratomalacie die partielle Unterernährung eine wesentliche Rolle spielt.

\section{Literaturverzeichnis.}

1) Holst und Frölich, Biochem. Zeitschr. Bd. XLIII. S. 89. 1912.

2) Freise, Monatssehr. f. Kinderheilk. Bd. XII. Abt. Orig. S. 687. 1914.

3) Eykman, Virch. Arch. Bd. CXIVIII. S. 523.

4) Hopkins Journ. of physiol. XLIV. p. 425.

5) Osborne and Mendel, Zeitschr. f. phys. Chemie. Bd. LXXX. S. 307. 1912.

6) Stepp, Biochem. Zeitschr. Bd.XXXII.S. 452; Zeitschr, f. Biol. Bd. LVII. S. 135.

7) Funk, Journ. of physiol, XLIII u. XLV. 1911 u. 1912.

8) Suzuki, Shimamura und Odake, Biochem. Zeitschr. Bd. XLIII. S. 89. 1912.

9) Förster, Graefe-Saemisch. Bd. XVII, 1. S. 225.

10) Dötsch, v. Graefe's Arch. f. Ophth. Bd. XLIX. S. 405. 1900.

11) Thalberg, Arch. f. Augenheilk. Bd. XII. S. 315. 1883.

12) Hirota, Zentralbl. f. inn. Med. 1898. S. 385.

13) Erdman n, Klin. Monatsbl. f. Augenheilk. 1914. S. 520. 